\section{(2) OPEN ACCESS}

\title{
The role of perceived discrimination in predicting changes in health behaviours among African Americans in the Jackson Heart Study
}

\author{
Allana T. Forde $\odot,{ }^{1,2}$ Mario Sims, ${ }^{3}$ Xu Wang, ${ }^{4}$ Sharrelle Barber, ${ }^{2}$ Ana V. Diez Roux ${ }^{2}$
}

\begin{abstract}
- Additional supplemental material is published online only. To view, please visit the journal online (http://dx. doi.org/10.1136/jech-2020215998)
\end{abstract}

${ }^{1}$ Division of Intramural Research, National Institute on Minority Health and Health Disparities, National Institutes of Health, Bethesda, Maryland, USA ${ }^{2}$ The Urban Health Collaborative, and the Department of Epidemiology and Biostatistics, Drexel University Dornsife School of Public Health, Philadelphia, Pennsylvania, USA ${ }^{3}$ Department of Medicine, University of Mississippi Medical Center, Jackson, Mississippi, USA ${ }^{4}$ Department of Biostatistics, University of Washington, Seattle, Washington, USA

\section{Correspondence to}

Dr Allana T. Forde, Division of Intramural Research, National Institute on Minority Health and Health Disparities, National Institutes of Health, Bethesda MD 20892-0001, USA; allana.forde@nih.gov

Received 8 November 2020 Accepted 22 May 2021

\begin{abstract}
Background This study examined whether perceived discrimination was associated with health behaviours over time and whether associations of discrimination with behaviours varied by attribution of discrimination. Methods Multinomial logistic regression was used to estimate ORs and Cls for the associations of discrimination (everyday, lifetime, stress from lifetime discrimination) with health behaviours (cigarette smoking, alcohol use) over time among 3050 African Americans in the Jackson Heart Study from visit 1 (2000-2004) to visit 3 (2009-2013). Smoking status was classified as persistent current, persistent former, persistent never, current to former and former/never to current smokers. Alcohol use status was classified as persistent heavy, persistent moderate/none, heavy to moderate/none and moderate/none to heavy alcohol users.
\end{abstract}

Results Higher everyday discrimination was associated with persistent current smoking (OR per SD higher discrimination 1.26, 95\% Cl 1.11,1.43) and with persistent former smoking (high vs low OR $1.32,95 \% \mathrm{Cl}$ $1.02,1.70)$ relative to persistent never smoking. Similar findings were observed for lifetime discrimination and persistent current smoking (high vs low OR 1.85, 95\% Cl $1.15,2.95$ ) and with persistent former smoking (high vs low OR 1.45, 95\% Cl 1.06, 1.98). Participants reporting lifetime discrimination as very stressful compared with not stressful were more likely to be persistent former smokers (OR 1.44, 95\% Cl 1.04,1.99). Associations did not vary by discrimination attribution.

Conclusion Discrimination did not predict changes in smoking status or alcohol use. Discrimination was associated with persistent current smoking status, which may provide a plausible mechanism through which discrimination impacts the health of African Americans.

\section{INTRODUCTION}

African Americans have a higher risk of cardiovascular disease $(\mathrm{CVD})^{1-3}$ compared with other racial and ethnic groups in the USA. ${ }^{34}$ Racial discrimination has been hypothesised to contribute to these well-documented racial inequities. In support of this hypothesis, studies have linked perceived discrimination to subclinical $\mathrm{CVD}^{5}$ and to CVD events. ${ }^{6}$ However, questions remain regarding the specific mechanisms that may explain these associations.

One plausible mechanism through which discrimination may impact cardiovascular health is via the stress pathway, whereby stress arising from discrimination activates the sympathetic nervous system and hypothalamic-pituitary-adrenal axis. $^{78}$ Another potential mechanism linking discrimination to cardiovascular health is through maladaptive coping behaviours in response to stress, ${ }^{9}$ such as smoking $^{10-12}$ and alcohol use. ${ }^{13} 14$

There is evidence to suggest that African Americans may engage in maladaptive behaviours as one way to buffer or reduce the chronic stress that results from discrimination. ${ }^{1516}$ Maladaptive coping behaviours in response to discrimination are further compounded by targeted marketing of tobacco and alcohol products within African American communities, ${ }^{17}$ and may contribute to the disproportionate burden of chronic illness among African Americans. Chronic illnesses occur disproportionately among African Americans, which may be a consequence of engaging in such behaviours. ${ }^{16} 18$

Several studies have reported associations of discrimination with smoking and alcohol use. $^{12}$ 19-23 However, these studies have been mostly cross-sectional, limiting causal inferences. In addition, they have not explored the moderating role of discrimination attribution in the association between discrimination and health behaviours. Discrimination attributed to race has been posited to be a more intense form of discrimination affecting African Americans. ${ }^{24}$ Thus, the impact of discrimination on health behaviours may differ among those attributing discrimination to race and those attributing discrimination to other reasons. ${ }^{19}$

The Jackson Heart Study (JHS) provides an opportunity to address these gaps in our understanding of the links between discrimination and health behaviours. We used data from the JHS to examine the extent to which multiple measures of discrimination (everyday, lifetime) and stress from lifetime discrimination are associated with changes in health behaviours (smoking and alcohol) over time, and whether discrimination attribution modifies these associations. Based on the plausible mechanisms linking discrimination to smoking and alcohol use, we hypothesised that high levels of discrimination would be associated with the persistence of smoking and alcohol use over time and with the uptake of these behaviours (eg, from never to current). We further hypothesised that the appraisal of lifetime discrimination as stressful would also lead to persistence or uptake of unhealthy behaviours. 


\section{METHODS}

\section{The JHS cohort}

The JHS is a community-based, prospective cohort study that was designed to examine CVD among non-institutionalised African American adults (21-95 years of age), who live in the tricounty area of Jackson, Mississippi (Hinds, Madison, Rankin counties). Participants were sampled from four recruitment pools at baseline: (1) Jackson participants of the Atherosclerosis Risk in Communities study (30\%); (2) participants randomly sampled from the Mississippi Department of Transportation Driver's License and Identification List (17\%); (3) volunteers who signed up for the study (22\%); and (4) family members of participants who agreed to be a part of the study (31\%). Additional details about the design and recruitment of participants are described elsewhere. ${ }^{25-27}$

Information on demographics, health behaviours, CVD risk factors and psychosocial factors (including discrimination) was obtained from self-administered questionnaires, in-home interviews and clinical examinations across three waves of data collection (2000-2013). ${ }^{2528} \mathrm{JHS}$ participants were enrolled $(\mathrm{n}=5306)$ during baseline, henceforth referred to as visit 1 (2000-2004). Additional data were collected during the follow-up assessments at visit 2 (2005-2008) and visit 3 (2009-2013). ${ }^{25} 29$ Follow-up rates were $79.2 \%$ from visit 1 to visit $2(n=4205)$ and $90.8 \%$ from visit 2 to visit $3(\mathrm{n}=3819)$. A fourth wave of data collection is ongoing.

\section{Measures}

Discrimination

Two measures of discrimination, everyday and lifetime, were obtained at visit 1 . Everyday discrimination was adapted from Williams' Everyday Discrimination Scale (Cronbach's $\alpha=0.88$ ) to capture how often on a day-to-day basis participants experienced unfair treatment in their everyday lives within nine domains $(e g, . .$. treated with less courtesy..., people act as if you are dishonest). Responses ranged from 1 ('never') to 7 ('several times a day') ${ }^{30}$ (online supplemental table S1). Everyday discrimination was examined as a continuous variable by taking the mean of the response scores to each of the nine questions and converted to SD units. Based on these responses, we also created tertiles of everyday discrimination.

Lifetime discrimination was based on Nancy Krieger's Lifetime Discrimination Scale (Cronbach's $\alpha=0.78)^{30}$ and captured the lifetime occurrence (yes/no) of unfair treatment experienced by participants across nine domains (eg, at work, at school, getting a job) (online supplemental table S1). Due to the skewed distribution of lifetime discrimination values, lifetime discrimination was not treated as a continuous variable. Based on previous literature, ${ }^{31}$ we summed the responses for which unfair treatment was reported across the nine domains (ranging from 0 to 9$),{ }^{32} 33$ and created three categories of lifetime discrimination (low: no discrimination (0), medium: values $<$ median value (range from 1 to 2 ), high: values $\geq$ median value (range from 3 to 9)) to address the skewed distribution of the lifetime discrimination values.

Perceived stress that derives from lifetime discrimination (or appraisal) ${ }^{30}$ was also examined as a separate exposure among participants who reported at least one experience of lifetime discrimination ${ }^{78}$ (online supplemental table S1).

Participants who reported experiencing discrimination were also asked to answer a single question indicating the main reason for experiences of discrimination (separate questions for everyday discrimination and lifetime discrimination).
Predetermined response options for discrimination attribution were age, sex, race, height, weight or some other reason. Based on prior work, two categories of discrimination attribution (racial vs all non-racial factors combined) were created from the predetermined attribution responses. ${ }^{19} 31$

\section{Health behaviours}

Outcomes included self-reported smoking and alcohol use that were collected from interviewer-administered questionnaires at visit 1 (2000-2004) and visit 3 (2009-2013). No health behaviour information was available for visit 2 (2005-2008). Using data from the two visits, we created a single variable for each person and for each of the two behaviours that captured change in that behaviour between visit 1 and visit 3 .

\section{Smoking}

At visit 1, cigarette smoking status was based on two questions from the Tobacco Use form: (1) 'Have you smoked at least 400 cigarettes in your lifetime?' and (2) 'Do you now smoke cigarettes? 34 Based on the responses to these two questions, we created three categories to define cigarette smoking status at visit 1: (1) current smoker ( $>400$ cigarettes in the lifetime and currently smoking); (2) former smoker ( $>400$ cigarettes in the lifetime and currently not smoking), and (3) never smoker ( $\leq 400$ cigarettes in the lifetime). The smoking question at visit 3 was slightly different. At visit 3 , participants were asked about whether they ever used any tobacco products regularly (including cigars, or cigarillos, pipes, chewing tobacco, or snuff/ dip) in the past 12 months ('In the past 12 months have you ever regularly used a tobacco product?') and asked separately about the number of cigarettes smoked per day, cigars smoked per week, pipefuls of pipes smoked per week, tobacco chewed per week and cans of dip/snuff used per week. Using the responses to these two questions we created two categories to define cigarette smoking status at visit 3: (1) current smoker (regular use of a tobacco product in the past 12 months and smoked $\geq 1$ cigarette per day in the past 12 months) and (2) not current smoker (no regular use of a tobacco product in the past 12 months or 0 cigarettes smoked per day in the past 12 months).

Based on previous work, ${ }^{35}$ we classified change in cigarette smoking status from visit 1 to visit 3 into five groups: (1) persistent current: 'current' at visit 1 and 'current' at visit 3; (2) persistent former: 'former' at visit 1 and 'not current' at visit 3; (3) persistent never: 'never' at visit 1 and 'not current' at visit 3 ; (4) current to former (improved): 'current' at visit 1 and 'not current' at visit 3; and (5) former/never to current (worsened): 'former' or 'never' at visit 1 and 'current' at visit 3 .

\section{Alcohol use}

At visit 1 and visit 3, alcohol use was defined per the responses to the following questions asked in the Alcohol and Drug Use questionnaire: In the past 12 months, (1) Have you ever consumed an alcoholic beverage? and (2) On average, on the days that you drank alcohol, how many drinks (eg, 12-ounce beer, a 4-ounce glass of wine or an ounce of liquor) did you have a day? Based on the responses to these two questions, we used the Centers for Disease Control and Prevention classification to create three categories of alcohol use: (1) heavy drinking (consumed $>1$ drink per day for women, consumed $>2$ drinks per day for men), (2) moderate drinking (consumed 1 drink per day for women and consumed 1-2 drinks per day for men), and (3) none (consumed 0 drinks per day or never consumed an alcoholic beverage or stopped drinking alcohol more than 1 year ago). ${ }^{36}$ 


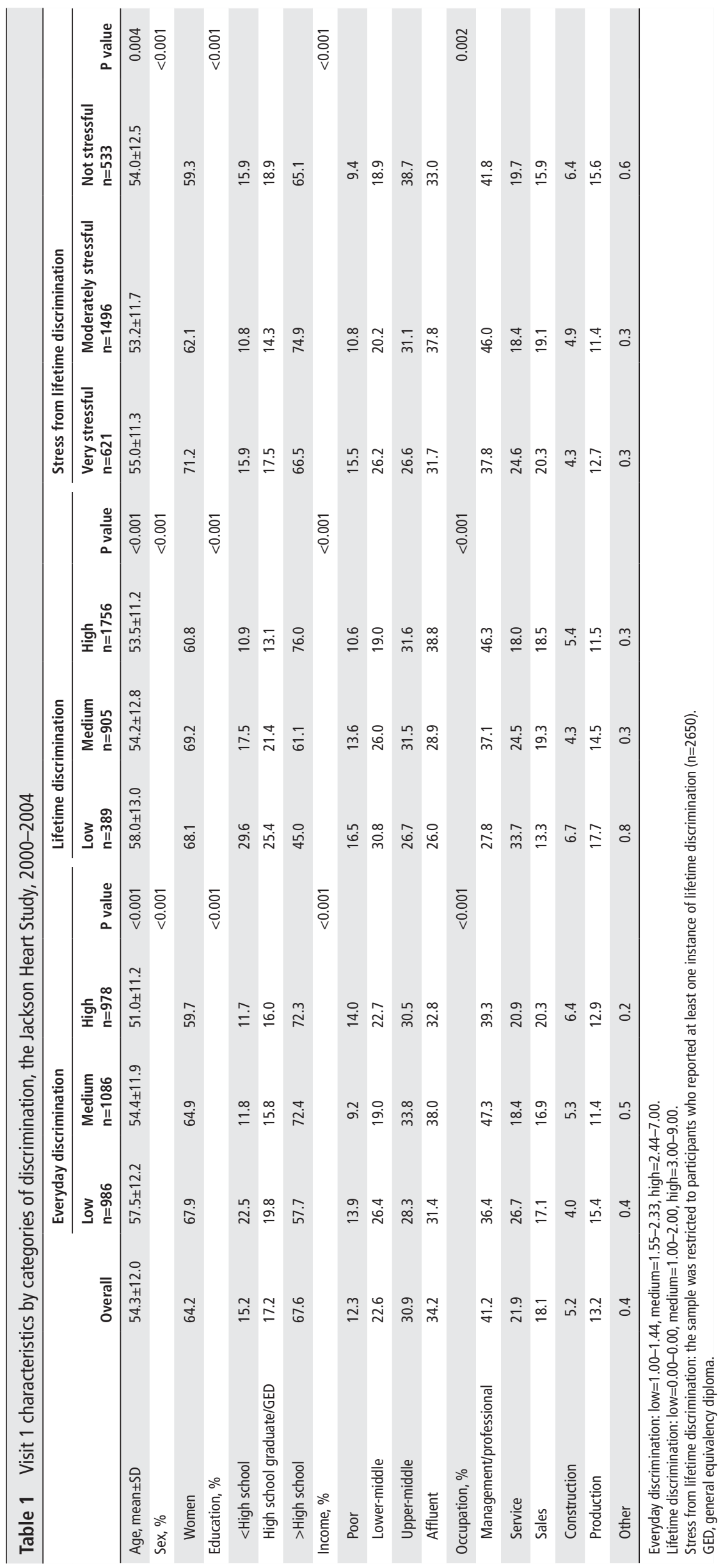




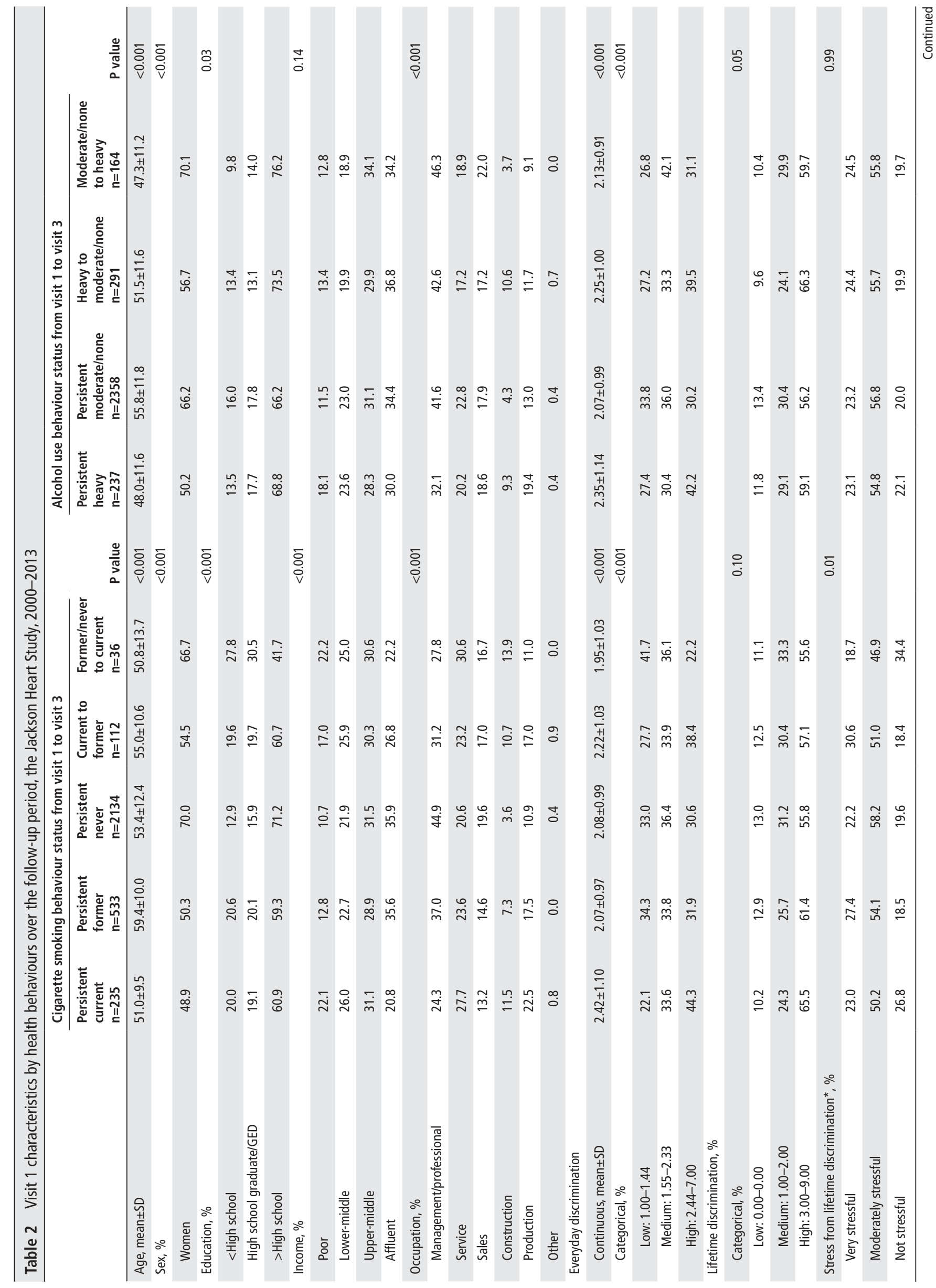




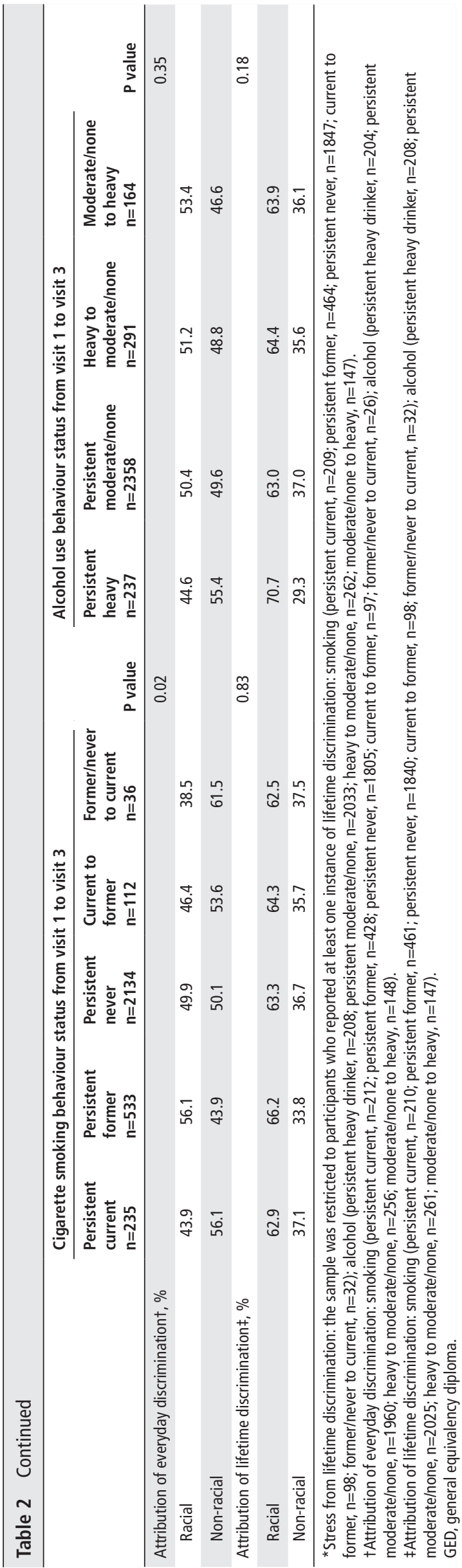

Based on prior work, ${ }^{35}$ we classified change in alcohol use status into four groups: (1) persistent heavy: 'heavy' at visit 1 and 'heavy' at visit 3; (2) persistent moderate/none: 'moderate' or 'none' at visit 1 and 'moderate' or' none' at visit 3; (3) heavy to moderate/none (improved): 'heavy' at visit 1 and 'moderate' or 'none' at visit 3; and (4) moderate/none to heavy (worsened): 'moderate' or 'none' at visit 1 and 'heavy' at visit 3 .

\section{Covariates}

Baseline covariates included age (continuous), sex (men, women) and socioeconomic status (SES, captured by education, income and occupation). Self-reported educational attainment was classified into three categories: (1) less than high school diploma; (2) high school graduate or general equivalency diploma; and (3) vocational school, trade school or college graduate. Income that was based on family income, family size and poverty level was classified as (1) poor; (2) lower-middle; (3) upper-middle; and (4) affluent. Occupation was coded as: (1) management/professional; (2) service; (3) sales; (4) construction; (5) production; and (6) other (farming, fishing, forestry, military, sick, unemployed, retired, other). We conceptualised discrimination would lead to health behaviours, which would then lead to chronic health conditions. Therefore, we did not include chronic health conditions as covariates in the models.

\section{Statistical analysis}

Of the 5306 JHS participants, 3819 were eligible to be included in our study because they completed both visits 1 and 3 . We excluded 117 participants who had missing information on either alcohol or smoking status at visit 1 or visit 3, an additional 144 who had missing information on everyday or lifetime discrimination at visit 1 and another 508 were excluded because they had missing information on SES at visit 1. Participants who completed visits 1 and 3 but were excluded from our analyses $(n=769)$, were as likely as the participants who were included in our final analyses $(n=3050)$ to experience discrimination (lifetime, everyday), smoke cigarettes and consume alcohol.

The distribution of the visit 1 characteristics of the study population were examined across categories of discrimination and health behaviours. Differences in the distribution were tested using $\chi^{2}$ tests or t-tests and described using percentages for categorical variables and means with SDs for continuous variables. Multinomial logistic regression was used to estimate ORs and CIs for the associations of dimensions of discrimination (everyday, lifetime, stress from lifetime discrimination) with the between-visit (2000-2013) status change in smoking and alcohol, sequentially adjusting for age, sex and SES variables. Specifically, the multinomial logistic regression modelled the categorical status change variable (a single measure per participant; five categories for smoking and four categories for alcohol use) as the outcome and the baseline discrimination score at visit 1 as the exposure. Model 1 was not adjusted for any other variables; model 2 adjusted for age and sex; and model 3 adjusted for age, sex and SES. Interaction product terms were included in the models to test the statistical significance of effect modification by discrimination attribution. Results were considered statistically significant if the probability value ( $p$ value) was $<0.05$. Analyses were performed using Statistical Analysis Software (SAS) version 9.4 (SAS Institute Inc, Cary, NC).

\section{RESULTS}

The sample was 64\% women, 68\% college educated, 34\% affluent and $41 \%$ had a management/professional occupation. 


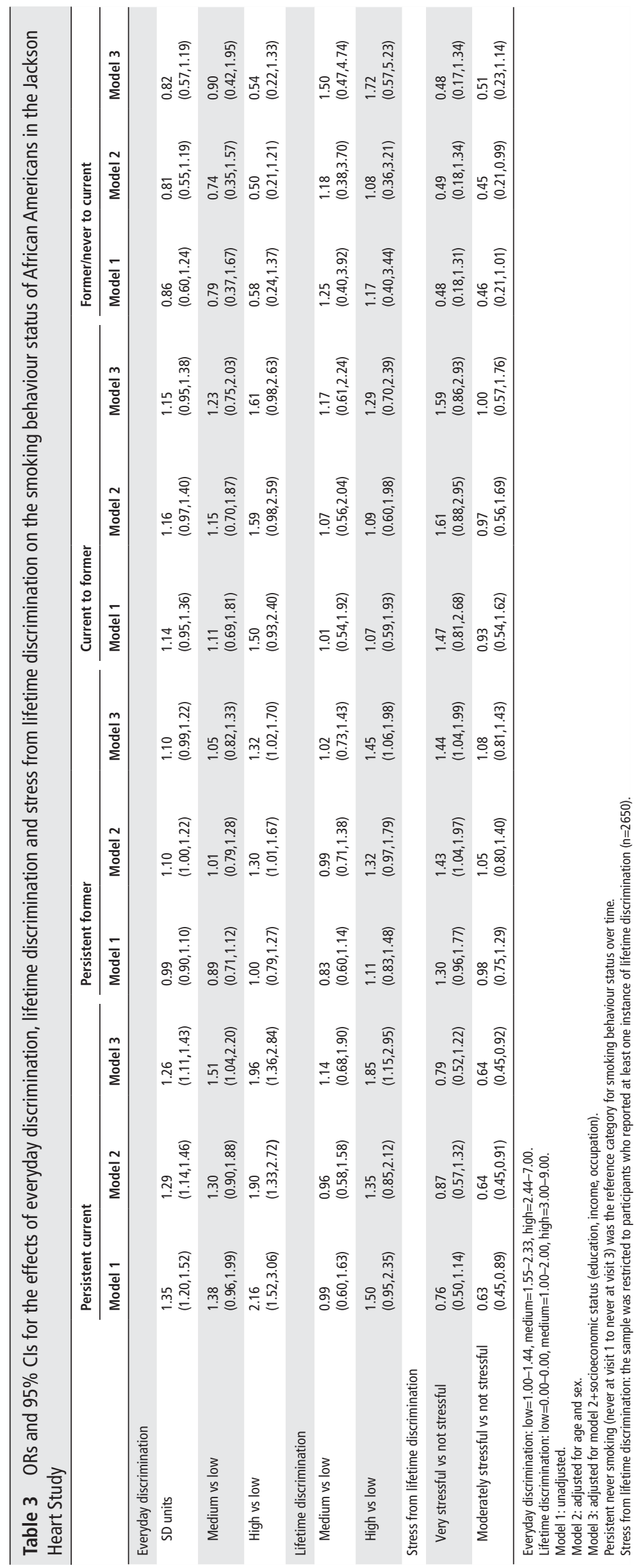




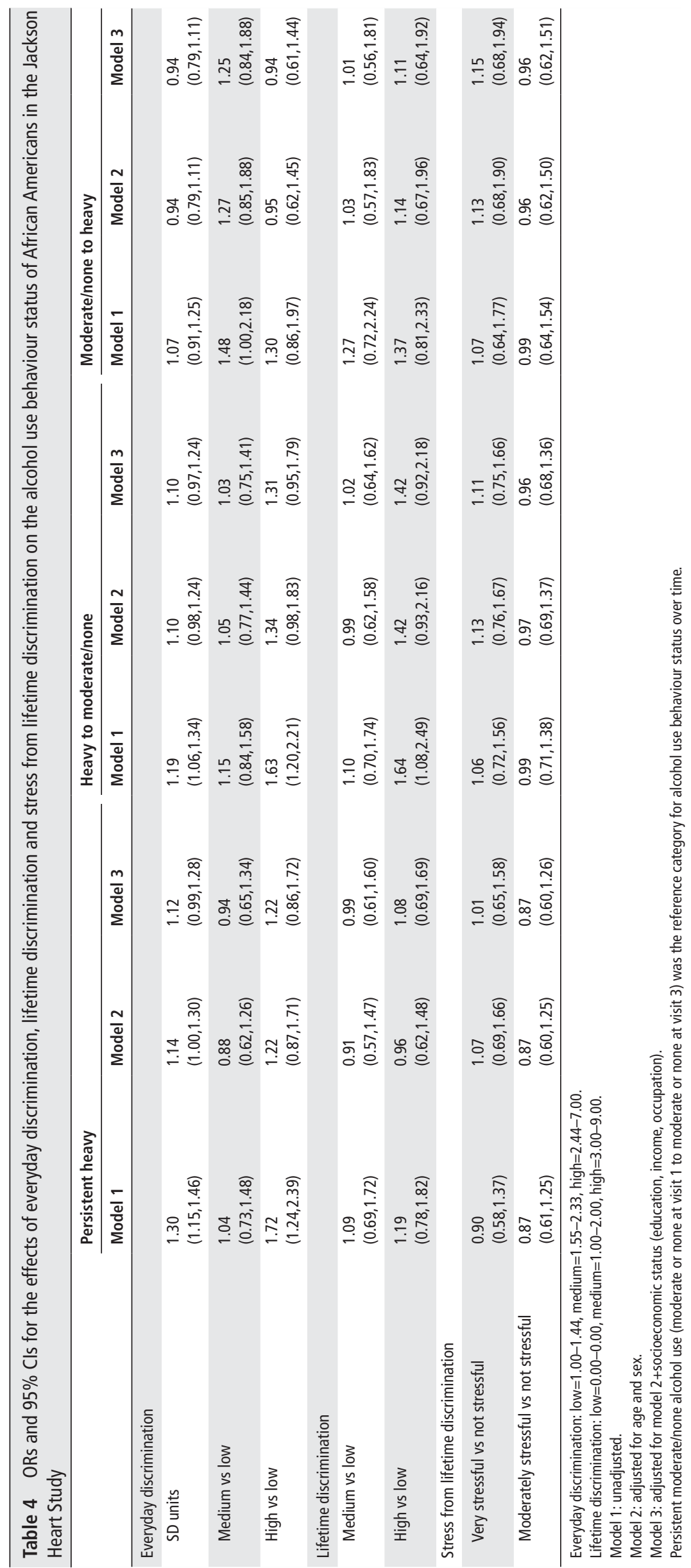


Participants reporting high everyday discrimination were more likely to be younger, men, college educated and had higher income than those reporting low everyday discrimination. Those reporting high levels of lifetime discrimination were more likely to be younger and men (table 1).

Eight percent of participants were persistent current smokers across both visits, $17 \%$ remained former smokers, $70 \%$ remained never smokers, $4 \%$ had improved smoking status (current to former) and 1\% had a worsened smoking status (former or never to current) (table 2). Persistent current smokers were more likely to be younger, men, less college educated and had lower income compared with those who maintained their status as never smokers. Persistent current smokers also reported higher levels of everyday and lifetime discrimination compared with persistent never smokers (table 2).

Eight percent of participants were persistent heavy alcohol drinkers across both visits, 77\% were persistent moderate or nonusers of alcohol at both visits, $10 \%$ improved their alcohol use (heavy to moderate or none) and 5\% developed worse alcohol use behaviour (moderate or none to heavy) (table 2). Participants who were persistent heavy alcohol users were younger and more educated than those who engaged in moderate alcohol use/ abstained from alcohol over the follow-up period. They were also more likely than persistent moderate users/non-users of alcohol to experience high levels of discrimination (everyday, lifetime) (table 2).

In models adjusted for age, sex and SES, everyday discrimination was associated with higher odds of being a persistent current smoker in a graded fashion (OR for tertiles of score: medium vs low OR 1.51, 95\% CI 1.04,2.20; high vs low OR 1.96, 95\% CI 1.36,2.84). Each SD higher score was associated with $26 \%$ higher odds of being a persistent current smoker (OR 1.26, 95\% CI 1.11,1.43). Being in the highest tertile of everyday discrimination was also associated with a higher odds of being a persistent former smoker (high vs low OR 1.32, 95\% CI 1.02,1.70) relative to persistent never smokers (table 3, model 3).

Lifetime discrimination was also associated with higher odds of being a persistent current smoker (high vs low OR 1.85, 95\% CI $1.15,2.95$ ) and being a persistent former smoker (high vs low OR $1.45,95 \%$ CI 1.06,1.98) relative to persistent never smokers after adjustment for age, sex and SES (table 3, model 3).

Participants reporting lifetime discrimination as very stressful compared with not stressful were also more likely to be persistent former smokers (OR 1.44, 95\% CI 1.04,1.99) relative to persistent never smokers (table 3, model 3). Everyday discrimination and lifetime discrimination were not associated with changes in alcohol use or persistent alcohol use (table 4, model 3).

Discrimination attribution did not modify the associations between discrimination and health behaviours (smoking status and alcohol use) over time for everyday discrimination ( $\mathrm{p}$ value for interaction: smoking, $p=0.38$; alcohol, $p=0.67$ ), lifetime discrimination ( $\mathrm{p}$ value for interaction: smoking, $\mathrm{p}=0.97$; alcohol, $\mathrm{p}=0.91$ ) or stress derived from lifetime discrimination ( $\mathrm{p}$ value for interaction: smoking, $\mathrm{p}=0.21$; alcohol, $\mathrm{p}=0.43$ ).

\section{DISCUSSION}

Most studies investigating discrimination and health behaviours have been cross-sectional and few have included multiple dimensions of discrimination. Our study is the only study, to our knowledge, to examine the associations of discrimination with change in cigarette smoking status and alcohol use over time in a large sample of African American adults. In our study, everyday discrimination, lifetime discrimination and stress from lifetime discrimination were not associated with changes in smoking status or alcohol use. High levels of everyday and lifetime discrimination were, however, associated with being a persistent current smoker and with being a persistent former smoker. High stress derived from lifetime discrimination was also associated with being a persistent former smoker.

Our findings for persistent current smoking status are consistent with previous cross-sectional studies that observed a positive association between lifetime discrimination and current smoking among African Americans in the JHS (only among women, regardless of discrimination attribution) ${ }^{19}$ Coronary Artery Risk Development in Young Adults ${ }^{2037}$ and the Multi-Ethnic Study of Atherosclerosis. ${ }^{21}$ The only study ${ }^{19}$ to include everyday discrimination also reported a positive association of everyday discrimination with current smoking among African American men and women in the JHS (regardless of discrimination attribution). Unlike previous work showing that reports of racial discrimination as extremely stressful were associated with higher rates of smoking, ${ }^{22}$ we found no evidence that stress from lifetime discrimination was associated with persistent current smoking, but the sample size $(n=209)$ in this group was relatively small.

Findings for alcohol use have been mixed, with some studies reporting an association between lifetime discrimination and increased alcohol use, ${ }^{202137}$ but one study reporting no evidence of an association between workplace discrimination and heavy drinking. ${ }^{38}$ Everyday discrimination, lifetime discrimination and stress from lifetime discrimination were not associated with changes in alcohol use, which may be due to the lower prevalence of alcohol use observed in the JHS or to measurement error in alcohol use, which is known to be difficult to measure.

There is evidence to suggest that stress associated with discrimination causes individuals to become more vulnerable to depression, anxiety disorder and psychological distress, which can lead to cigarette smoking ${ }^{12}$ and alcohol use. ${ }^{39}$ It has also been hypothesised that African Americans may engage in unhealthy behaviours to cope with the elevated stress arising from discrimination. ${ }^{40}$ Indeed, this hypothesis has been put forward to explain the paradox by which some mental health outcomes are better in African Americans than White Americans, but physical health outcomes show the opposite pattern. ${ }^{40}$ Our results are consistent with an impact of stress from discrimination on smoking as demonstrated by the association of discrimination with persistent smoking. While we hypothesised that cigarette smoking and alcohol use were strategies used to cope with stress from discrimination, we were unable to explicitly test whether the participants engaged in maladaptive behaviours to reduce stress from discrimination.

Several limitations of the data should be considered when interpreting our findings. The sample only included African American adults residing in Jackson, Mississippi, which limited the generalisability of our findings to African Americans in other regions. Discrimination was analysed at one point in time, which prevented us from examining the impact of timevarying discrimination on health behaviours over time. Due to lack of information on the timing of exposure to discrimination, it was not possible to examine the lag time between exposure to discrimination and occurrence of health behaviours. While the overall sample size was adequate in our study, there was limited power to detect significant associations between discrimination and changes in smoking status due to small numbers in the group of former or never smokers at visit 1 who changed to current smokers at visit 3. Similarly, sample size may have limited our ability to detect effect modification by attribution 
of discrimination. Additional follow-up of the JHS will allow extension of these analyses, yielding more power.

Residual confounding was also a possibility because of the lack of data on episodic or binge drinking, as well as data on availability of tobacco and alcohol products. In addition, the results may be biased due to the inclusion of participants who were more educated and had a higher income than those who were excluded from our study. Our study benefited from the use of multiple measures of discrimination, a large sample of African American adults, a heterogeneous population, the long follow-up period and the longitudinal study design which allowed for the examination of changes in smoking and alcohol use status.

Our study expands on previous studies to highlight the impact of discrimination on persistent current smoking status. We show that experiences of discrimination may result in persistent smoking. Importantly, stress resulting from these interpersonal experiences may affect smoking by also interacting with other manifestations of structural racism at different levels including living in stressful neighbourhood environments, experiencing stressful jobs and the targeting of tobacco marketing to African American communities, among other factors. The impact of structural racism on health thus needs to be examined using measures of racism (and its consequences) at multiple levels. Our study adds to growing evidence on the many ways in which structural racism affects the health of African Americans.

\section{What is already known on this subject}

- Previous studies have reported associations between perceived discrimination and adverse health behaviours. However, most studies have been cross-sectional.

\section{What this study adds}

- This study examined the associations of multiple measures of discrimination (everyday, lifetime) with changes in health behaviours over time in a large population-based cohort of African Americans. We found that everyday discrimination and lifetime discrimination are related to persistent smoking among African Americans. These results further highlight the mechanisms through which discrimination affects the health of African Americans.

Acknowledgements The authors wish to thank the staff and participants of the Jackson Heart Study.

Contributors ATF contributed to the conception and design of the work, the analyses, the interpretation of the data and writing of the manuscript. MS contributed to the study design and provided his expertise related to the Jackson Heart Study data. XW provided additional guidance regarding the statistical analyses and interpretation of the data. SB contributed to the conceptualisation of the links between discrimination and health behaviours content in the manuscript. AVDR directed the overall study. All authors provided critical feedback on each version of the manuscript and approved the final manuscript.

Funding The Jackson Heart Study is supported and conducted in collaboration with Jackson State University (HHSN268201800013I), Tougaloo College (HHSN268201800014I), the Mississippi State Department of Health (HHSN268201800015I) and the University of Mississippi Medical Center (HHSN268201800010I, HHSN268201800011I and HHSN268201800012I) contracts from the National Heart, Lung, and Blood Institute (NHLBI) and the National Institute on Minority Health and Health Disparities (NIMHD). Additional support was provided by grant R01HL117323 from the NHLBI. ATF is supported by the Division of Intramural Research, NIMHD, National Institutes of Health. MS is supported by the JHS contract and grant R01HL135200 from the NHLBI and grant 15SFDRN26140001 from the American Heart Association.
Disclaimer The views expressed in this manuscript are those of the authors and do not necessarily represent the views of the National Heart, Lung, and Blood Institute; the National Institute on Minority Health and Health Disparities (NIMHD); the National Institutes of Health; or the U.S. Department of Health and Human Services.

Competing interests None declared.

Patient consent for publication Not required.

Ethics approval The JHS protocol was approved by the Institutional Review Boards of Jackson State University, the University of Mississippi Medical Center and Tougaloo College. Written informed consent was obtained from all participants.

Provenance and peer review Not commissioned; externally peer reviewed.

Data availability statement The JHS data are available to researchers with approved manuscript proposals. The JHS data and materials can be requested from the JHS Committee at https://www.jacksonheartstudy.org/Research/Study-Data/ Data-Access

Supplemental material This content has been supplied by the author(s). It has not been vetted by BMJ Publishing Group Limited (BMJ) and may not have been peer-reviewed. Any opinions or recommendations discussed are solely those of the author(s) and are not endorsed by BMJ. BMJ disclaims all liability and responsibility arising from any reliance placed on the content. Where the content includes any translated material, BMJ does not warrant the accuracy and reliability of the translations (including but not limited to local regulations, clinical guidelines, terminology, drug names and drug dosages), and is not responsible for any error and/or omissions arising from translation and adaptation or otherwise.

Open access This is an open access article distributed in accordance with the Creative Commons Attribution Non Commercial (CC BY-NC 4.0) license, which permits others to distribute, remix, adapt, build upon this work non-commercially, and license their derivative works on different terms, provided the original work is properly cited, appropriate credit is given, any changes made indicated, and the use is non-commercial. See: http://creativecommons.org/licenses/by-nc/4.0/.

ORCID iD

Allana T. Forde http://orcid.org/0000-0002-3097-0038

\section{REFERENCES}

1 Williams DR. Miles to go before we sleep: racial inequities in health. J Health Soc Behav 2012;53): :279-95

2 Krieger N. Embodying inequality: epidemiologic perspectives. Baywood Publishing Company Inc, 2005. https://kriya2.kriyadocs.com/review_content/?doi=heartjnl-2019316442\&customer=bmi\&project=heartjnl

3 Carnethon MR, Pu J, Howard G, et al. Cardiovascular health in African Americans: a scientific statement from the American heart association. Circulation 2017:136:e393-423

4 Graham G. Disparities in cardiovascular disease risk in the United States. Curr Cardiol Rev 2015:11:238-45.

5 Lewis TT, Williams DR, Tamene M, et al. Self-reported experiences of discrimination and cardiovascular disease. Curr Cardiovasc Risk Rep 2014:8:365.

6 Everson-Rose SA, Lutsey PL, Roetker NS, et al. Perceived discrimination and incident cardiovascular events: the multi-ethnic study of atherosclerosis. Am J Epidemiol 2015;182:225-34.

7 Williams DR, Mohammed SA. Discrimination and racial disparities in health: evidence and needed research. J Behav Med 2009;32:20-47.

8 Sims M, Diez-Roux AV, Dudley A, et al. Perceived discrimination and hypertension among African Americans in the Jackson heart study. Am J Public Health 2012;102(Suppl 2):S258-65

9 Brondolo E, Gallo LC, Myers HF. Race, racism and health: disparities, mechanisms, and interventions. J Behav Med 2009;32:1-8.

10 Bennett GG, Wolin KY, Robinson EL, et al. Perceived racial/ethnic harassment and tobacco use among African American young adults. Am J Public Health 2005:95:238-40.

11 Landrine H, Klonoff EA, Corral I, et al. Conceptualizing and measuring ethnic discrimination in health research. J Behav Med 2006:29:79-94.

12 Purnell JQ, Peppone LJ, Alcaraz K, et al. Perceived discrimination, psychological distress, and current smoking status: results from the behavioral risk factor surveillance system reactions to race module, 2004-2008. Am J Public Health 2012:102:844-51.

13 Williams DR, Neighbors HW, Jackson JS. Racial/Ethnic discrimination and health: findings from community studies. Am J Public Health 2003;93:200-8.

14 Martin JK, Tuch SA, Roman PM. Problem drinking patterns among African Americans: the impacts of reports of discrimination, perceptions of prejudice, and "risky" coping strategies. J Health Soc Behav 2003:44:408-25.

15 Jackson JS, Knight KM. Race and self-regulatory health behaviors: the role of the stress response and the HPA axis in physical and mental health disparities. In: Social structures, aging, and self-regulation in the elderly, 2006: 189-239. 
16 Pascoe EA, Smart Richman L. Perceived discrimination and health: a meta-analytic review. Psychol Bull 2009;135:531-54.

17 Lee JGL, Henriksen L, Rose SW, et al. A systematic review of neighborhood disparities in point-of-sale tobacco marketing. Am J Public Health 2015;105:e8-18.

18 Sims M, Lipford KJ, Patel N, et al. Psychosocial factors and behaviors in African Americans: the Jackson heart study. Am J Prev Med 2017;52:S48-55.

19 Sims M, Diez-Roux AV, Gebreab SY, et al. Perceived discrimination is associated with health behaviors among African-Americans in the Jackson heart study. J Epidemiol Community Health 2016;70:187-94.

20 Borrell LN, Kiefe Cl, Diez-Roux AV, et al. Racial discrimination, racial/ethnic segregation, and health behaviors in the cardia study. Ethn Health 2013;18:227-43.

21 Borrell LN, Diez Roux AV, Jacobs DR, et al. Perceived racial/ethnic discrimination, smoking and alcohol consumption in the multi-ethnic study of atherosclerosis (MESA). Prev Med 2010;51:307-12.

22 Landrine $\mathrm{H}$, Klonoff EA. Racial discrimination and cigarette smoking among blacks: findings from two studies. Ethn Dis 2000;10:195-202.

23 Wade J, Peralta RL. Perceived racial discrimination, heavy episodic drinking, and alcohol abstinence among African American and White college students. J Ethn Subst Abuse 2017:16:165-80.

24 Lewis TT, Cogburn CD, Williams DR. Self-reported experiences of discrimination and health: scientific advances, ongoing controversies, and emerging issues. Annu Rev Clin Psychol 2015;11:407-40.

25 Fuqua SR, Wyatt SB, Andrew ME, et al. Recruiting African-American research participation in the Jackson heart study: methods, response rates, and sample description. Ethn Dis 2005;15:S6-18.

26 Payne TJ, Wyatt SB, Mosley TH, et al. Sociocultural methods in the Jackson heart study: conceptual and descriptive overview. Ethn Dis 2005;15:S6-38.

27 Taylor HA, Wilson JG, Jones DW, et al. Toward resolution of cardiovascular health disparities in African Americans: design and methods of the Jackson heart study. Ethn Dis 2005;15:S6-4.

28 Dolezsar CM, McGrath JJ, Herzig AJM, et al. Perceived racial discrimination and hypertension: a comprehensive systematic review. Health Psychol 2014;33:20-34.
29 Wilson Jet al. The Jackson heart study: an overview. Ethn Dis 2005;15:S6.

30 Sims M, Wyatt SB, Gutierrez ML, et al. Development and psychometric testing of a multidimensional instrument of perceived discrimination among African Americans in the Jackson heart study. Ethn Dis 2009;19:56.

31 Forde AT, Sims M, Muntner P, et al. Discrimination and hypertension risk among African Americans in the Jackson heart study. Hypertension 2020;76:715-23.

32 Krieger N, Sidney S. Racial discrimination and blood pressure: the cardia study of young black and white adults. Am J Public Health 1996;86:1370-8.

33 Krieger N. Racial and gender discrimination: risk factors for high blood pressure? SoC Sci Med 1990;30:1273-81.

34 Howard G, Manolio TA, Burke GL, et al. Does the association of risk factors and atherosclerosis change with age? An analysis of the combined ARIC and CHS cohorts. The Atherosclerosis risk in communities (ARIC) and cardiovascular health study (CHS) Investigators. Stroke 1997;28:1693-701.

35 Wang $X$, Auchincloss AH, Barber S, et al. Neighborhood social environment as risk factors to health behavior among African Americans: the Jackson heart study. Health Place 2017;45: :199-207.

36 Committee, U.S.D.G.A. Dietary guidelines for Americans, 2010. US Department of Health and Human Services, US Department of Agriculture, 2010.

37 Borrell LN, Jacobs DR, Williams DR, et al. Self-reported racial discrimination and substance use in the coronary artery risk development in adults study. Am J Epidemiol 2007;166:1068-79.

38 Yen IH, Ragland DR, Greiner BA, et al. Workplace discrimination and alcohol consumption: findings from the San Francisco Munl health and safety study. Ethn Dis 1999:9:70-80

39 Hatzenbuehler ML, Corbin WR, Fromme K. Discrimination and alcohol-related problems among college students: a prospective examination of mediating effects. Drug Alcohol Depend 2011;115:213-20.

40 Jackson JS, Knight KM, Rafferty JA. Race and unhealthy behaviors: chronic stress, the HPA axis, and physical and mental health disparities over the life course. Am J Public Health 2010;100:933-9. 